\title{
Segmental infantile hemangioma and concomitant hypertension in three African American neonates
}

Sara A. Kullberg BA, Michelle N. Rheault MD, Anita Haggstrom MD, Sheilagh M. Maguiness MD

1University of Minnesota Medical School, Minneapolis, Minnesota

2Department of Pediatrics, University of Minnesota, Minneapolis, Minnesota

3Department of Dermatology and Pediatrics, Indiana University, Indianapolis, Indiana

4Department of Dermatology and Pediatrics, University of Minnesota, Minneapolis, Minnesota

\begin{abstract}
We present three African American infants with segmental, ulcerated infantile hemangiomas and concomitant, persistent hypertension. When treated with beta-blocker therapy, the hemangiomas decreased in size and the ulcerations resolved, but there was no impact on the elevated blood pressure in one of our patients. We failed to identify any associations between infantile hemangioma and hypertension in the literature.
\end{abstract}

Keywords: infantile hemangioma, neonatal hypertension, PHACE syndrome

This is the author's manuscript of the work published in final edited form as:

Kullberg, S. A., Rheault, M. N., Haggstrom, A., \& Maguiness, S. M. (2020). Segmental infantile hemangioma and concomitant hypertension in three African American neonates. Pediatric Dermatology, 37(3), 524-526. https://doi.org/10.1111/pde.14119 


\section{Segmental infantile hemangioma and concomitant hypertension in three}

\section{African American neonates}

\section{Introduction}

Infantile hemangiomas $(\mathrm{IH})$ proliferate over the first weeks of life; with systemic beta-blockers, they can regress significantly, most resolving within 6 months.1, 2 Hypertension (HTN) is rare in the first year of life in term infants and can be associated with various pathologies, commonly renovascular or renoparenchymal in origin. Neonatal HTN requires further investigation with echocardiography, renal imaging, and laboratory testing to determine the underlying etiology.3 We describe three African American infants with segmental IH and HTN at diagnosis.

\section{Case 1}

A 7-week-old, full-term, African American girl with Dandy-Walker variant per prenatal ultrasound presented with worsening left forehead and eyelid discoloration involving most of the left face, lip, and neck (Figure 1A,B). PHACE evaluation was notable for posterior fossa abnormalities by MRI/MRA (vermian hypoplasia, pontomedullary hypoplasia, and many cisterna magna), cerebral arterial anomalies (anomalous superior orbital artery, left A1 and M1 segment saccular aneurysms, multilobulated left posterior cerebral artery aneurysm, and vascular arcade along left perimesencephalic cistern), as well as a morning glory disk malformation of the left eye. Underlying risk for stroke was assessed as intermediate. She was referred to pediatric neurology and started aspirin $40 \mathrm{mg}$ daily.4 Her manual blood pressure, taken from the upper extremity while calm, was $102 / 56 \mathrm{~mm} \mathrm{Hg}$ at initial presentation. As the facial $\mathrm{IH}$ demonstrated ocular involvement and superficial ulceration, propranolol was initiated. Over the course of her hospitalization, blood pressures remained persistently elevated despite initiation of propranolol in the range of 106-119/48-72 mm Hg $(95 \%<105 / 68 \mathrm{~mm} \mathrm{Hg}) .3$ Family history was notable for HTN in the father; the mother did not have a diagnosis of known HTN before or during her pregnancy. Comprehensive work-up for HTN was initiated. Laboratory investigations (renin/aldosterone, TSH/T4, 
renal panel, and urinalysis) and imaging, including renal ultrasound, aortic/IVC/iliac ultrasound, and echocardiogram, were all unremarkable. After discharge, propranolol was titrated up to full dose $(2 \mathrm{mg} / \mathrm{kg} / \mathrm{d})$ and blood pressures remained elevated until about 3 months of age. She remained on propranolol at 16 months of age with normal blood pressures.

\section{Case 2}

A 2-month-old, full-term, African American boy presented with worsening discoloration of the lower lip, left lower cheek, and left upper chest with decreased food intake. Examination showed a large, telangiectatic, vascular plaque consistent with IH on the left mandible, with partial S3/S4 distribution and ulceration of the lower lip. PHACE work-up was negative. Blood pressure was $123 / 81 \mathrm{~mm} \mathrm{Hg}$ at initial presentation (taken from the upper extremity manually while calm). HTN persisted with blood pressures 120-138/72-106 mm Hg over his initial 2-day hospitalization. Notably, there was no significant familial history of high blood pressure. Work-up for HTN including abdominal and aortic ultrasound, echocardiogram, urinalysis, C-reactive protein, renal panel, complete blood count, thyroid-stimulating hormone, and urine catecholamines (homovanillic acid and vanillylmandelic acid) were unremarkable. He was treated with propranolol, $2 \mathrm{mg} / \mathrm{kg} / \mathrm{d}$ divided three times daily. The lip ulceration resolved within 3 weeks. Blood pressures normalized within 1 week of discharge. Blood pressure remained normal at 14 months of age on propranolol.

\section{Case 3}

A 3-month-old, African American girl presented with a vascular plaque consistent with segmental IH on the left forearm extending from her elbow to her wrist, which developed a cobblestone appearance and subsequently ulcerated. She was born at 24 weeks gestation with pregnancy complicated by preterm premature rupture of membranes, premature labor, and chorioamnionitis. Postnatally, she experienced apnea of prematurity, respiratory failure, and grade 1 intracranial hemorrhage. This $\mathrm{IH}$ was treated with wound care and pulsed dye laser shortly after consultation. The ulceration was recalcitrant, and she 
received a total of four treatments of pulsed dye laser between the ages of 3-8 months of age. Notably, hypertension was diagnosed at 3 weeks of age and was initially managed with amlodipine, though substituted with atenolol after her dermatology evaluation. Echocardiogram was unremarkable, and renal ultrasound demonstrated grade 1 hydronephrosis, deemed non-contributory. No family history of HTN was found. Atenolol was eventually discontinued at 18 months old, without rebound of IH or recurrence of HTN.

\section{Discussion}

Normal blood pressure in infants varies by age and prematurity. Normal blood pressures in term infants in the first year of life are generally considered $<105 / 68 \mathrm{~mm} \mathrm{Hg.3} \mathrm{Though} \mathrm{uncommon} \mathrm{in} \mathrm{healthy} \mathrm{term}$ infants, HTN can be seen in up to $0.2 \%-3 \%$ of term or preterm neonates admitted to the NICU.5 Our three patients' BPs were in the hypertensive range at the time of presentation and, in our first case, persisted despite propranolol initiation. This was particular surprising for HTN to persist in our infant diagnosed with PHACE syndrome, as previous literature has demonstrated concern for hypotension with the use of propranolol to treat IHs given their associated congenital arterial anomalies, which can lead to peripheral ischemia and ulceration, or reduced cerebral blood flow and neurologic consequences.6

Neonatal hypertension can result from a variety of potential etiologies, the three most common being umbilical artery thrombi or emboli, aortic coarctation, and bronchopulmonary dysplasia. Additional causes for neonatal and infantile hypertension not to be overlooked include underlying renovascular disease and renal congenital malformations, post-natal acute kidney injury, post-natal corticosteroid administration, and maternal history of hypertension.7

To evaluate for hypertension in infancy, a thorough history including previous prenatal/perinatal exposures, prenatal imaging, and medications is recommended. A complete physical examination is also necessary. This includes evaluating general appearance for assessment of related syndromes, assessing neurologic status, abdominal examination to evaluate for masses, genitourinary examination for 
anomalies and virilization, and cardiac examination including four extremity blood pressures to screen for aortic coarctation.3, 7 Initial laboratory work-up should include trending of blood pressures, assessment of kidney function (blood urea nitrogen, creatinine, and electrolytes), and urinalysis, as well as consideration of pertinent hormone levels (cortisone, renin/aldosterone, and thyroxine). Imaging studies should additionally be performed, including chest X-ray echocardiography, and aortic and/or renal ultrasound depending on level of clinical suspicion. 3,8

Given our infants were evaluated in academic tertiary care centers with comprehensive subspecialty care, recommended examinations and studies were performed as recommended by pediatric nephrology but failed to identify an etiology for the HTN in these infants. In clinical practice, the co-occurrence of IH or PHACE patients experiencing HTN due to their vascular anomalies has not been reported. In our patients, pain or discomfort may have been a precipitating factor for hypertension at presentation; however, this would not explain the continued hypertension in the first patient case. The exact mechanism for how the congenital arterial anomalies found in PHACE syndrome or even the aberrant blood vessel architecture and endothelial cell proliferation characterized in IHs could potentially lead to an increase in blood pressure has not been specifically studied. Physiologically, it is well known that slight structural alterations within the arterial lumen leading to seemingly insignificant reductions in radius can result in significant changes in arterial resistance and therefore increases in blood pressure. 9

Previous studies have assessed racial variation for the prevalence of HTN and IH, as well as beta-blocker sensitivity. A previous multicenter cohort study of over 2100 enrolled neonates demonstrated an increased risk of HTN in Caucasian but not African American neonates born between 30 and 35 weeks gestation.5 These results conflict with another multicenter cohort study of over 200 premature neonates, which determined non-white race as a significant risk factor for eventual adolescent hypertension.10 While IHs can present in all patients of all ethnicities, they are much more common in Caucasians and less common in African American populations.11 Thus, the finding that all patients in this series are African American is unusual. One hypothesis for the racial discrepancy of IH prevalence is thought to be due to increased 
secretion of fibromodulin secreted by melanocytes. Fibromodulin is a key regulator of angiogenesis, which could effectively protect African American patients with increased melanocyte activity from angiogenesis-dependent diseases like IH and age-related macular degeneration.12 Additionally, even when controlled for diet, gender, and BMI, previous studies demonstrate that beta-blockers appear to be less effective in African American versus white subjects in modifying both BP and heart rate.13 Despite these findings, all three infants responded well to treatment with beta-blockers for their $\mathrm{IH}$, with reduction in volume, color, visual occlusion, and ulceration.

\section{CONCLUSION}

Segmental IHs presenting with concomitant HTN have not previously been reported. All IH patients with HTN warrant a comprehensive work-up. Evaluation with nephrology and cardiology may be appropriate in order to rule out known associations between segmental IHs and cardiac anomalies. In all three cases, hemangiomas improved on therapy. Blood pressures normalized for two of the infants during treatment with propranolol. Careful monitoring of blood pressure will be required when propranolol is discontinued. 


\section{References}

1. Léauté-Labrèze C, Hoeger P, Mazereeuw-Hautier J, et al. A randomized, controlled trial of oral propranolol in infantile hemangioma. N Engl J Med. 2015;372:735-746.

2. Drolet BA, Frommelt PC, Chamlin SL, et al. Initiation and use of propranolol for infantile hemangioma: report of a consensus conference. Pediatrics. 2013;131:128-140.

3. Dionne JM, Abitbol CL, Flynn JT. Hypertension in infancy: diagnosis, management and outcome. Pediatr Nephrol. 2012;27:17-32.

4. Garzon MC, Epstein LG, Heyer GL, et al. PHACE syndrome: consensus-derived diagnosis and care recommendations. J Pediatr. 2016;178:24-33.e2.

5. Kraut EJ, Boohaker LJ, Askenazi DJ, et al. Incidence of neonatal hypertension from a large multicenter study [Assessment Worldwide Acute Kidney Injury Epidemiology in Neonates - AWAKEN]. Pediatr Res. 2018;84:279-289.

6. Gnarra M, Solman L, Harper J, et al. Propranolol and prednisolone combination for the treatment of segmental haemangioma in PHACES syndrome. Br J Dermatol. 2015;173:242-246.

7. Sharma D, Farahbakhsh N, Shastri S, et al. Neonatal hypertension. J Matern Fetal Neonatal Med. 2017;30:540-550.

8. Flynn JT. Hypertension in the neonatal period. Curr Opin Pediatr. 2012;24:197-204.

9. Laurent S, Boutouyrie P. The structural factor of hypertension: large and small artery alterations. Circ Res. 2015;116:1007-1021.

10. Vohr BR, Allan W, Katz KH, et al. Early predictors of hypertension in prematurely born adolescents. Acta Paediatr. 2010;99:1812-1818. 
11. Cordisco MR. Hemangiomas of infancy: epidemiology. In: Mattassi R, Loose DA, Vaghi M, eds. Hemangiomas and Vascular Malformations. Milano: Springer; 2009.

https://doi.org/10.1155/2012/645678.

12. Adini I, Ghosh K, Adini A, et al. Melanocyte-secreted fibromodulin promotes an angiogenic microenvironment. J Clin Invest. 2014;124:425-436.

13. Kurnik D, Li C, Sofowora GG, et al. Beta-1-Adrenoceptor genetic variants and ethnicity independently affect response to beta-blockade. Pharmacogenet Genomics. 2008;18:895-902. 
(A)

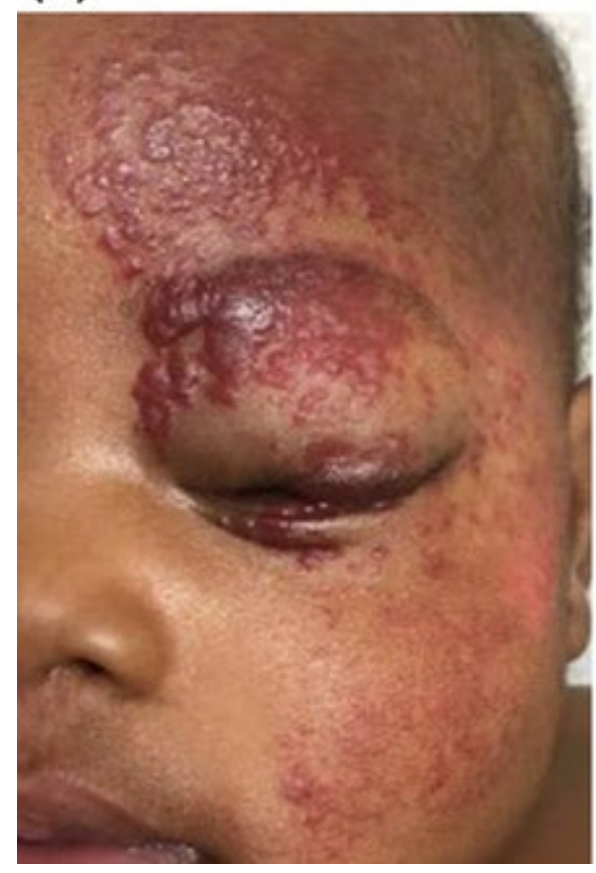

(B)

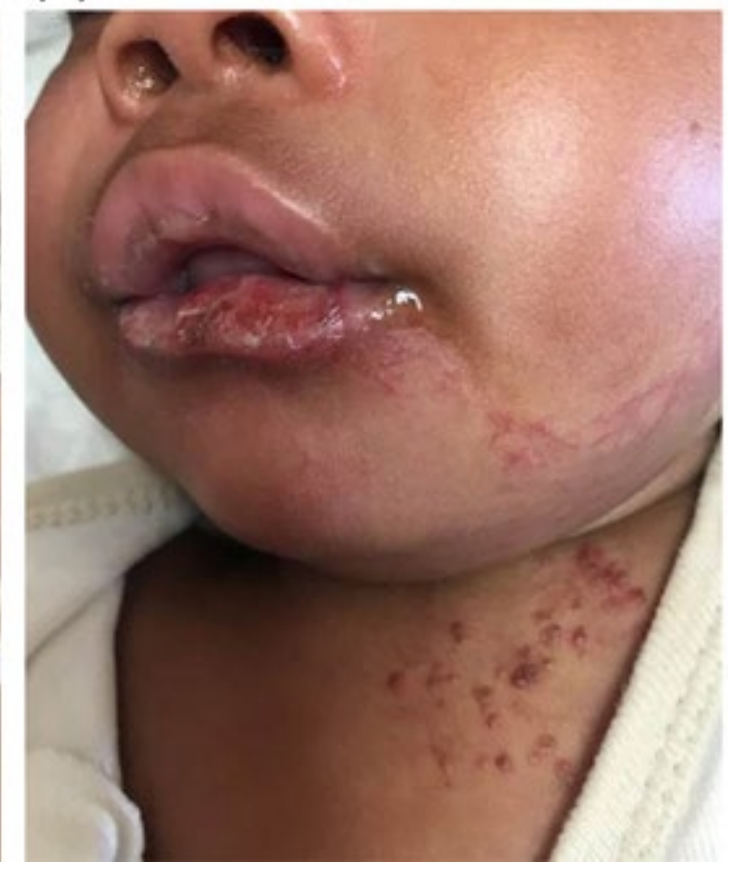

Figure 1

A, B, Case 1 Segmental infantile hemangioma of the face and neck in African American girl with PHACE syndrome 